\title{
B-type natriuretic peptide levels predict extent and severity of coronary disease in non-ST elevation coronary syndromes and normal left ventricular systolic function
}

\author{
Alberto Palazzuoli ${ }^{\text {a, }}$, Alan Maisel ${ }^{\text {c }}$, Maria Caputo ${ }^{\text {a }}$, Massimo Fineschi ${ }^{\text {b }}$, Ilaria Quatrini ${ }^{\text {a }}$, Anna Calabrò ${ }^{\text {a }}$ \\ Maria Stella Campagna ${ }^{a}$, Beatrice Franci ${ }^{a}$, Stefanie Grothgar ${ }^{a}$, Carlo Pierli ${ }^{b}$, Ranuccio Nuti ${ }^{a}$ \\ a Department of Internal Medicine and Metabolic Disease Cardiology Section, University of Siena, Italy \\ b U.O. Invasive Cardiology Le Scotte Hospital Siena, Italy \\ c Cardiac Care Unit and Heart Failure Program, San Diego Veterans Affairs Medical Center, San Diego, CA, USA
}

\section{A R T I C L E I N F O}

\section{Article history:}

Received 8 September 2010

Received in revised form 8 December 2010

Accepted 27 December 2010

Available online 13 January 2011

\section{Keywords:}

B-type natriuretic peptide

Coronary artery disease

Coronary angiography

Risk stratification

\begin{abstract}
A B S T R A C T
Background: B-type natriuretic peptide (BNP) has been used recently as a biological marker in patients with coronary artery disease (CAD) with ST-elevation, as well as without ST-elevation. BNP is able to predict systolic dysfunction, adding new prognostic information to existing traditional markers. However is not known if there is a relation between the quantity of BNP levels and the severity of coronary artery disease. Methods: This study compared B-type natriuretic peptide (BNP) levels in patients with stable angina (SA) and acute coronary syndromes (ACS) without ST-elevation in relation to angiographic lesions using TIMI and Gensini Scores. We studied 282 patients with CAD without ST elevation and preserved systolic function. BNP samples were measured in all recruited patients within 24 hours of hospitalization.

Results: BNP values were progressively increased in relation to the severity of diagnosis: SA $(52.6 \pm 49.4 \mathrm{pg} / \mathrm{mL}) \mathrm{UA}$ $(243.3 \pm 212 \mathrm{pg} / \mathrm{mL})$ NSTE-ACS $(421.7 \pm 334 \mathrm{pg} / \mathrm{mL}) \quad(p<0.0001$ and $p<0.007$ respectively). No statistically significant difference was observed between patients with SA and controls $(21.2 \pm 6.8 \mathrm{pg} / \mathrm{mL})$. The analysis of BNP levels in relation to the number of involved vessels demonstrated significantly increased levels in patients with multivessel disease compared to patients with 1 or 2 vessel disease $(1-86.2 \pm 46.3 \mathrm{pg} / \mathrm{mL} ; 2-127 \pm 297 \mathrm{pg} / \mathrm{mL}$; $3-295 \pm 318 \mathrm{pg} / \mathrm{mL} ; 4-297 \pm 347 \mathrm{pg} / \mathrm{mL} p<0.001$ and $p<0.003)$. Evaluation of BNP using Gensini Score showed a strong relation between BNP and coronary disease extension $(r=0.38 p<0.0001)$.This trend was maintained in all CAD groups $(\mathrm{SA}=\mathrm{r} 0.54$; UA $r=0.36$ NSTE-ACS $r=0.28$ ).

Conclusions: Circulating BNP levels appear elevated in ACS with diffuse coronary involvement, even in the absence of systolic dysfunction. BNP is also associated with multi-vessel disease and the extension of coronary disease.
\end{abstract}

(c) 2011 Elsevier B.V. All rights reserved.

\section{Introduction}

B-type Natriuretic Peptide (BNP) is a peptide consisting of 32 aminoacids produced by the myocytes as a pro-hormone. It is released in response to ventricular dilatation and pressure overload, in its active form after peptidase degradation, into the cardiovascular system [1]. BNP levels are increased after myocardial infarction and high levels are related to an increased risk of adverse events [2]. Recently it has been demonstrated that BNP and N-terminal pro-B-type natriuretic peptide (NT pro-BNP) also provide predictive information on acute coronary syndromes (ACS) , and they appear related to the severity of CAD in patients affected with ACS $[3,4]$.

\footnotetext{
* Corresponding author. Department of Internal Medicine and Metabolic Diseases, Cardiology Section, Le Scotte Hospital, Viale Bracci 53100 Siena, Italy. Tel.: + 39 577585363, + 39 577585461; fax: + 39577233480 .

E-mail address: palazzuoli2@unisi.it (A. Palazzuoli).
}

Coronary syndromes without ST elevation are a heterogeneous disorder ranging from stable angina (SA) without evidence of myocardial necrosis, to myocardial infarction (NSTE-ACS). Because of different clinical presentations, coronary syndromes without ST elevation encompass a wide range of events and have different prognostic values in relation to the type of plaque lesions and the diffusion of coronary atherosclerosis. The risk assessment, based on clinical history and examination, electrocardiographic (ECG) characteristics, and markers of myocardial damage, still remains relatively inaccurate [5]. The risks of subsequent death and/or recurrent ischemic events also vary widely, depending on the presence of myocardial ischemic areas, irreversible myocyte injury, the hemodynamic consequences of ischemia and the extent of coronary atherosclerosis. Several studies in ACS patients demonstrated a strong association between increased BNP levels and poor clinical outcome; however, the underlying mechanisms responsible for this association are not well clarified: BNP and pro-BNP could be indicators for multivessel disease, poor TIMI flow, as well as markers of coronary disease extension $[4,6,7]$. To support this hypothesis, we compared BNP levels in patients 
with stable angina and ACS in relation to angiographic lesions, vessel narrowing, and the extension of coronary disease, using TIMI and Gensini Scores.

\section{Materials and methods}

\subsection{Study population}

The starting group consisted of 397 patients, 54 were excluded for left ventricular (LV) systolic dysfunction at echocardiographic examination, 40 for previous myocardial infarction or revascularization, and 21 for associated significant valvular disease. The study was performed on the remaining 282 subjects (154 males, 138 females) with the main complaint of chest pain and no-ST elevation, who underwent coronary artery angiography in our Department from May 2006 to October 2008. Patients were divided in four groups: Group 0 (controls, $n=32$ ) was submitted to angiographic examination for atypical chest pain, the angiography revealed healthy coronary vessels, Group I (stable angina SA, $n=71$ ), Group II (unstable angina UA, $n=83$ ), and Group III (non S-T elevation myocardial infarction NSTE-ACS, $n=96$ ).

SA was defined as typical angina that is precipitated by exertion or emotional stress, it is associated with an electrocardiographic (ECG) horizontal or downsloping S-T segment depression of more than $1.5 \mathrm{~mm}$ during exercise. These symptoms were combined with S-T segment depression and changes in biochemical biomarkers of myocardial necrosis (myoglobin CK-MB and Troponin TnT). SA was also diagnosed when the complete diagnostic protocol was performed in the Chest Pain Unit and did not demonstrate myocardial necrosis or ischemia. Patients who showed increased CKMB and/or troponin-I levels after the performance of percutaneous transluminal coronary angioplasty $(\mathrm{PCI})$ were not considered to have acute myocardial infarction (AMI).

UA is defined by ECG ST-segment depression or prominent T-wave inversion with negative biomarkers of necrosis in an appropriate clinical setting (chest discomfort or angina equivalent). Unstable angina was diagnosed when, in the absence of the above mentioned troponin-I or CKMB changes, suggestive chest pain lasting longer than $20 \mathrm{~min}$ was associated with either ST-segment depression $(\geq 0.1 \mathrm{mV})$ or T-wave inversion in the ECG, pre-discharge ischemic stress test, or significant coronary artery disease in an angiogram [8].

NSTE-ACS was diagnosed when an elevation of troponin-I levels ( $>1.0 \mathrm{ng} / \mathrm{mL}$ in any sample during the first 9 hours post-admission) and/or a typical CKMB curve occurred, with or without ST/T changes in the ECG, in the absence of any other demonstrable cause for the chest pain [9]. Patients were evaluated prior to blood sampling to determine their heart failure status according to the New York Heart Association. All recruited patients underwent echocardiographic examination (Hewlett-Packard, 5500 Sonos Philips) to evaluate modifications in parietal kinesis, left ventricular morphology and systolic function [10]. Blood samples for BNP were performed within 24 hours (median $14 \pm 6$ ) of hospital admission. Sixty milliliters of blood were taken from a peripheral veins and distributed in tubes for BNP detection. The study was approved by the Local Human Scientific Committee. All reported investigations have been carried out in accordance with the principles of the Declaration of Helsinki and written informed consent was obtained from all patients. Within the first 3 days of hospital admission, left cardiac catheterization and selective coronary angiography were performed.

\subsection{Exclusion criteria}

Patients with a history of myocardial infarction, valvular disease, acute or chronic heart failure, cardiomyopathy, systolic dysfunction, ejection fraction $<50 \%$, renal liver and neoplastic diseases were excluded. Patients with ST-elevations on the admission-electrocardiogram were excluded. Patients were also excluded when the evolution of the electrocardiogram showed the development of a new left bundle branch block or new Qwaves. Other exclusion criteria were known or suspected infectious or inflammatory conditions and scheduled revascularization procedures.

\subsection{Treatment protocol}

All patients underwent standard therapy with aspirin, nitrates, calcium-antagonists or beta-blockers, heparin or low molecular weight heparin. Patients with NSTE-ACS were also treated with glycoprotein IIb/IIIa inhibitors (Tirofiban) or clopidrogrel.

\subsection{Laboratory assays}

Creatine kinase levels were analyzed by a standard technique and its MB fractions by electrophoresis. Myoglobin and TnT were assayed by automate enzyme immunoassay. The levels of TnT were determined by a third-generation assay on an Elecsys (Roche Diagnostics, Basel, Switzerland), with the detection limit at $0.01 \mu \mathrm{g} / \mathrm{L}$ and a total coefficient of variance (CV) of $8 \%$ at $0.05 \mu \mathrm{g} / \mathrm{L}$ and $4.1 \%$ to $6.0 \%$ between 0.1 and $11 \mu \mathrm{g} / \mathrm{L}$. An excess of $0.05 \mu \mathrm{g} / \mathrm{l}$ for TnT and $50 \mu \mathrm{g} / \mathrm{L}$ for myoglobin were suggestive for significant myocardial damage. Plasma BNP was immediately analysed on the same EDTA-anti-coagulated blood sample collected on admission for CK-MB and troponin-I, using the quantitative immunofluorescence assay manufactured by Biosite (San Diego, CA, USA). The analytic sensitivity of the assay is $<5 \mathrm{pg} / \mathrm{mL}$ and the upper normal limit is considered to be $100 \mathrm{pg} / \mathrm{mL}$. Plasma CK-MB mass was measured by immunofluorescence assay manufactured by DadeBehring (Marburg, Germany). The analytic sensitivity of the assay is $0.6 \mathrm{ng} / \mathrm{mL}$ and the upper normal limit is considered to be $5.0 \mathrm{ng} / \mathrm{mL}$. Plasma troponin-I was measured by immunofluorescence assay manufactured by Dade-Behring. The analytic sensitivity of the assay is $0.1 \mathrm{ng} / \mathrm{mL}$ and the upper normal limit for the diagnosis of AMI was considered to be $1.0 \mathrm{ng} / \mathrm{mL}$.

\subsection{Angiographic analysis}

All coronary angiograms were evaluated at the TIMI angiographic score laboratory by physicians who were blinded to the patient's clinical or BNP status. Coronary stenosis was quantified using validated quantitative coronary angiography. Assessment of epicardial coronary flow was done using the TIMI flow grade according to previously described methods [11]. The Gensini score (GS) was used in the present study to assess the burden of coronary arteriosclerosis [12]. The GS system yields a qualitative and quantitative evaluation of the coronary angiogram; it grades the narrowing of the lumen of the coronary artery as 1 for $\leq 25 \%$ narrowing, 2 for $26-50 \%$ narrowing, 4 for $51-75 \%$ narrowing, 8 for $76-90 \%$ narrowing, 16 for $91-99 \%$ narrowing, and 32 for total occlusion. This primary score is multiplied by a factor that takes into account the importance of the position of the lesion in the coronary arterial tree ( 5 for the left main coronary artery, 2.5 for the proximal left anterior descending artery or proximal left circumflex artery and 1.5 for the mid-region, 1 for the distal left anterior descending artery, and 1 for the mid-distal region of the left circumflex artery or right coronary artery). In this study, the GS was expressed as the sum of the scores for all three coronary arteries in order to evaluate the entire extent of CAD. The grades of luminal narrowing were determined by the consensus opinion of two experienced interventional cardiologists.

\subsection{Statistical analysis}

The statistical analysis was performed using the SPSS software for Windows, version 9.0.1 (SPSS, Inc., Chicago, IL). Summary statistics for continuous variables were recorded as the mean $\pm S D$, and the categorical data was summarized as frequencies and percentages. Multiple group comparisons were performed using Kruskal-Wallis test followed by Mann-Whitney $U$ test when significant differences 
were detected. Correlations between continuous variables were calculated according to Spearman-Rho. Univariate logistic regression analysis was performed with the presence of CAD as the dependent variable and BNP levels, age, current smoking, cholesterol levels, diabetes, hypertension, body mass index (BMI) and ST segment deviation $>1 \mathrm{~mm}$, as independent variables, Odds Ratio (OR) is presented with 95\% confidence intervals. All numeric data are presented as the mean $\pm \mathrm{SD}$, and a $p$ value of less than 0.05 was considered statistically significant.

\section{Results}

\subsection{BNP and clinical presentation}

All of the remaining 282 patients had BNP levels and angiographic data: clinical characteristics and associated risk factor are reported in Table 1. BNP levels were similar in controls and the SA group (32.1 \pm $23 \mathrm{pg} / \mathrm{mL}$ vs $55.4 \pm 63 \mathrm{pg} / \mathrm{mL}$ NS) but it revealed progressively increasing values in relation to the diagnosis: BNP levels were significantly different between the SA Group and the UA Group $(237.1 \pm 261 \mathrm{pg} / \mathrm{mL} ; \mathrm{p}<0.01)$ as well as between the SA Group and the NSTE-ACS Group ( $340.2 \pm 283 \mathrm{pg} / \mathrm{mL} ; \mathrm{p}<0.001$ ). Even in ACS, BNP showed different values in the UA Group compared to the NSTEACS Group $(p<0.007)$ (Fig. 1). BNP $>80 \mathrm{pg} / \mathrm{mL}$ was recruited in 176 patients, most of whom had ACS: 21 had SA (12\%), 71 had UA (42\%), and 81 had NSTE-ACS (46\%). Multi-vessel disease was revealed more frequently in patients with BNP $>80 \mathrm{pg} / \mathrm{mL}$ (Table 2).

\subsection{BNP and coronary vessel involvement}

BNP was strictly related to the extent of coronary disease measured with traditional methods: significant progressive differences were assessed between the 1-vessel Group, the 2-vessel and 3-4-vessel Group (1-vessel 89.2 \pm 47.3 ; 2-vessels 221,8 $\pm 182,2$; 3 -4-vessels $295.8 \pm 264.5 \mathrm{pg} / \mathrm{mL}, p<0.001)$. A similar significant trend was found between the 2-vessel Group and the 3-vessel Group $(p<0.005)$, but not significant difference was detected between the 3-vessel and 4-vessel Group $(297.2 \pm 247 \mathrm{pg} / \mathrm{mL} \mathrm{NS})$ (Fig. 2). When the measurement was limited to the 1-vessel group $(n=44)$, we observed a significantly higher BNP in patients with left anterior descending artery (LAD) involvement than in patients with other coronary districts involvement $(121,4 \pm 56,5$ vs $78,6 \pm$ $64,2 \mathrm{pg} / \mathrm{mL}, p<0.05$ ).

The analysis of the Gensini Score (GS) demonstrated a strong correlation between coronary artery disease extension and BNP levels $(r=0.40, p<0.0001)$ (Fig. 3). This trend was maintained in all CAD Groups: SA GS 29.9 $\pm 20.5(r=0.55, p<0.0001)$; UA GS $42 \pm 20$ $(r=0.36, p<0.001)$; NSTE-ACS GS $46.4 \pm 17.2(r=0.28, p<0.05)$.

\subsection{BNP and risk factors}

We assessed the association of BNP levels with established cardiovascular risk factors, including hypercholesterolemia, arterial

Table 1

Clinical characteristics and risk factors associated in all group studied.

\begin{tabular}{llll}
\hline & Stable angina & Unstable angina & NSTEMI \\
\hline Number & 71 & 83 & 96 \\
Sex (m/f) & $40 / 31$ & $43 / 40$ & $58 / 38$ \\
Diabetes (n) & 17 & 40 & 42 \\
Hypertension (n) & 26 & 43 & 44 \\
Smoking (n) & 10 & 9 & 13 \\
Dyslipidemia (n) & 18 & 30 & 31 \\
S-T deviation & 8 & 55 & 72 \\
BNP (pg/ml) & $52.6 \pm 49.4$ & $243.3 \pm 212$ & $421.7 \pm 334$ \\
\hline
\end{tabular}

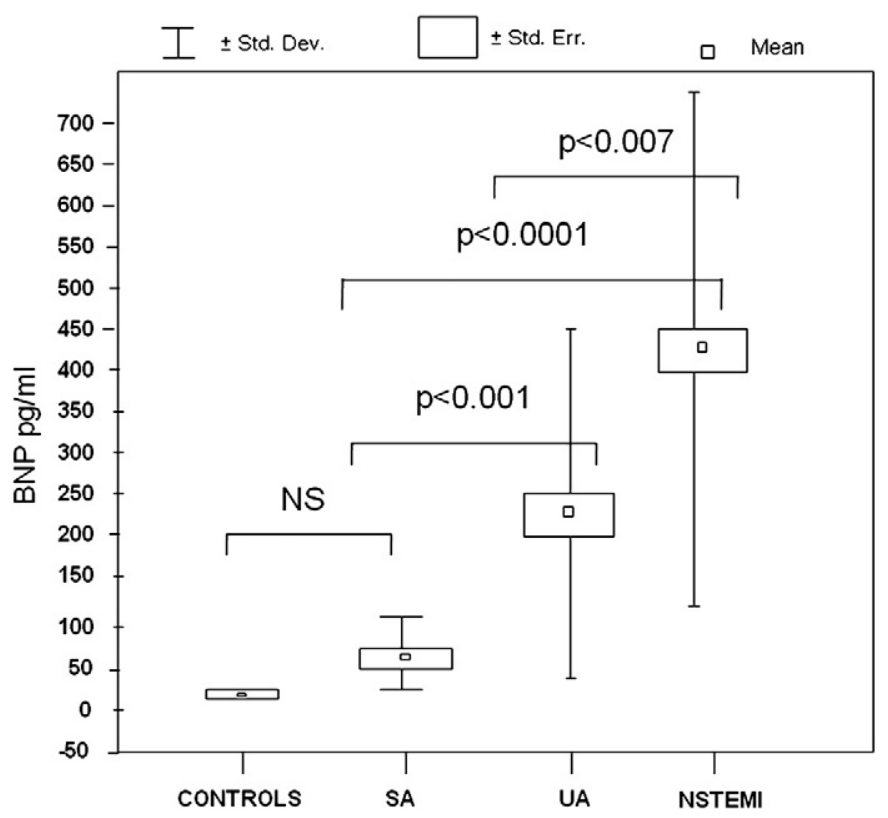

Fig. 1. BNP levels in Controls, Stable angina (SA), Unstable Angina ( UA), and Myocardial Infarction (NSTEMI).

hypertension, smoking, diabetes, and BMI. Notably, BNP was positively correlated to hypercholesterolemia $(r=0.22, p<0.01)$ and diabetes $(r=0.48, p<0.001)$. But there was no correlation with Body Mass Index (BMI), smoking, hypertension and gender. Univariate analysis showed that BNP levels were independently and positively associated with the presence of $\mathrm{CAD}(\mathrm{OR}=15.7, p<0.001)$; diabetes and ST deviation were associated with CAD ( OR 2.17, $p<0.05$ and OR $8.35, p<0.01$ respectively); age were weakly associated with CAD (OR $1.32, p<0.05$ ). However smoking, hypertension, and BMI did not show any association with the disease (Table 3 ).

\section{Discussion}

Many studies have shown that the elevation of BNP levels, as well as NT-proBNP levels, obtained after the acute phase in patients with a broad range of ACS independently predicts mortality. However, in most of the reports, authors did not distinguish between subjects with low ejection fraction and LV enlargement $[3,4,13]$. Because of its release in response to increased ventricular chamber pressure or wall tension, in ACS patients with decreased LV ejection fraction, elevated BNP levels reflect a high degree of myocardial dysfunction, with a higher risk of death and congestive heart failure.

The main finding of this study is that BNP levels are related not only to LV dysfunction but also to the severity of coronary atherosclerosis: patients with multi-vessel disease showed higher BNP levels than subjects with only one or two vessel involvement.

Table 2

Clinical characteristics and associated risk factors in patients with BNP $<80$ and $>80 \mathrm{pg} / \mathrm{ml}$.

\begin{tabular}{lll}
\hline & BNP $<80$ & BNP $>80$ \\
\hline Age (yrs) & $68.6 \pm 6.9$ & $72.5 \pm 8.1$ \\
Hypertension (\%) & 45.3 & 54.7 \\
Diabetes (\%) & 41.2 & 58.8 \\
Smoking (\%) & 70.7 & 29.3 \\
Dyslipidemia (\%) & 49.0 & 51.0 \\
SA & 57.9 & 11.9 \\
UA & 29.9 & 42.1 \\
NSTEMI & 12.2 & 46 \\
Multi-vessel disease (\%) & 28.7 & 71.3 \\
\hline
\end{tabular}




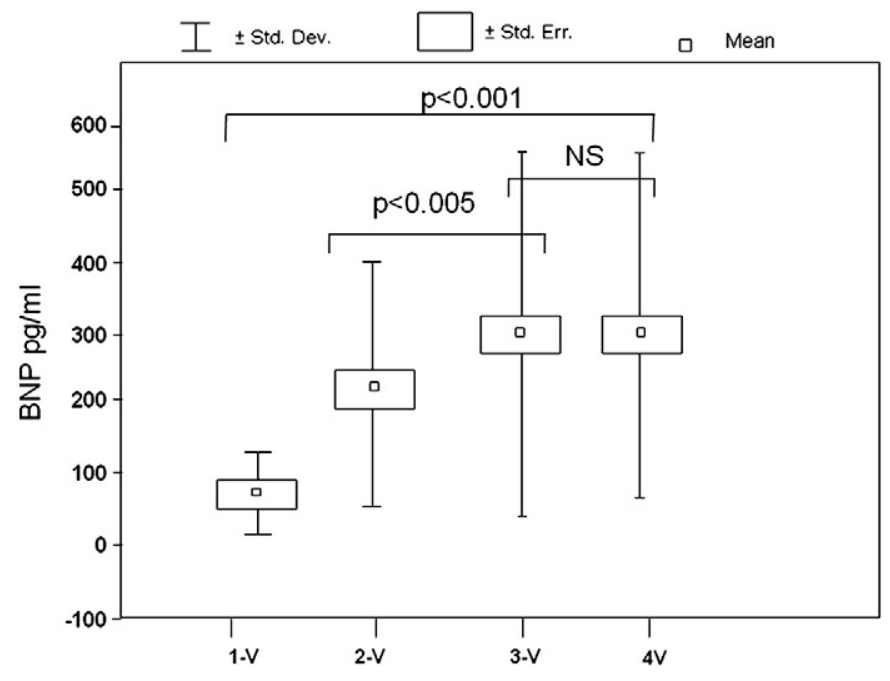

Fig. 2. BNP measurement in patients with mono-, bi- and multi-vessels disease. BNP levels progressively increase in patients with more diffuse coronary atherosclerosis.

This trend was confirmed independently of the diagnosis in all patient groups. Elevation of BNP levels appears strictly related to coronary artery disease. Indeed, none of the patients had LV systolic dysfunction and signs or symptoms of heart failure. Our results are in accordance with those of Sadanandan et al showing a correlation between TIMI flow, thigh of culprit stenosis and BNP levels [14]. However, with respect to the cited study, our sample was characterized by the absence of left ventricular dysfunction and enlargement which are the main factors responsible for BNP increase $[15,16]$. Again, we demonstrated that BNP threshold of $80 \mathrm{pg} / \mathrm{ml}$ appears able to predict the extension of coronary disease independently from LV systolic dysfunction and enlargement. The same value has been previously recognized as cutoff for mortality and risk for adverse events in patients with ACS $[3,4,14]$.

In a previous report, we confirmed this trend showing a direct correlation between BNP levels and prognosis, even in patients with preserved systolic function [17]. In this line, Weber et al. demon-

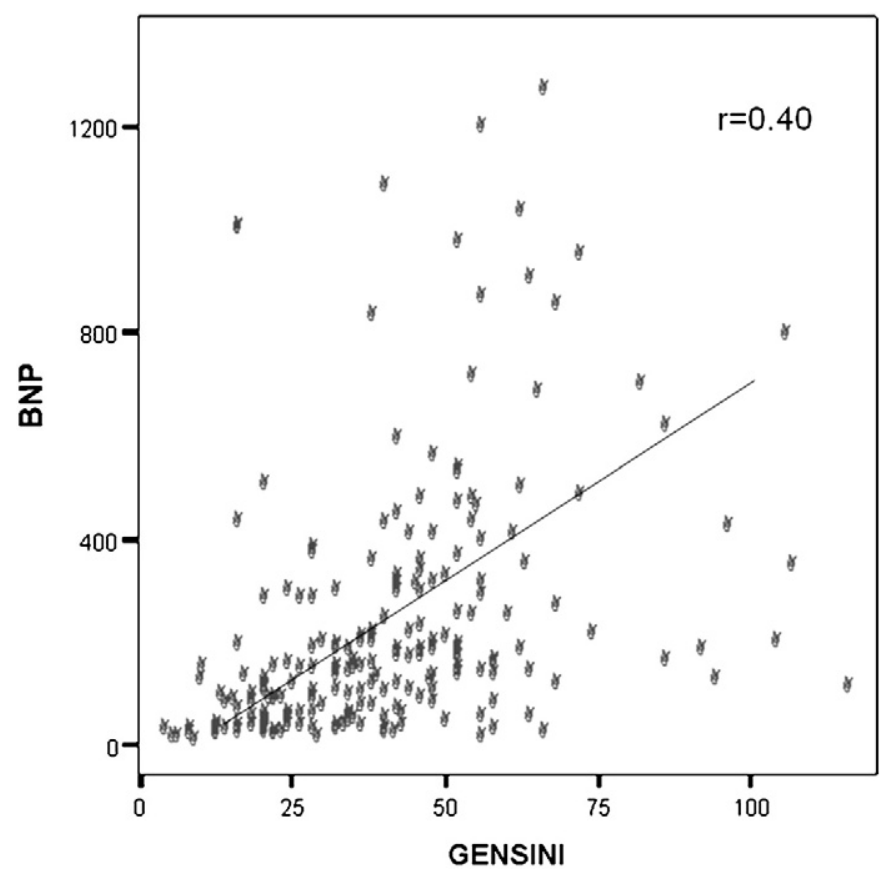

Fig. 3. Correlation between BNP and Gensini score in patients with CAD.
Table 3

Association of presence of CAD with BNP levels and traditional risk factors: BNP appears the most powerful indicator of CAD.

\begin{tabular}{lll}
\hline Variable & Pearson's correlation & Significance \\
\hline Age $>70$ yrs & 0.06 & $\mathrm{~ns}$ \\
Hypertension & 0.06 & $\mathrm{~ns}$ \\
Diabetes & 0.09 & $\mathrm{~ns}$ \\
Dyslipidemia & 0.07 & $\mathrm{~ns}$ \\
Smoking & 0.08 & $\mathrm{~ns}$ \\
CAD history & 0.18 & $<0.01$ \\
ST deviation $>2 \mathrm{~mm}$ & 0.46 & $<0.0001$ \\
Multi vessel disease & 0.20 & $<0.01$ \\
BNP $>80$ pg $/ \mathrm{ml}$ & 0.62 & $<0.0001$ \\
Gensini score $>20$ & 0.36 & $<0.0001$ \\
\hline
\end{tabular}

strated a close correlation between NT-proBNP and disease severity in patients with stable angina [18]. In contrast with the above data, the PEACE Trial showed a strong association between cardiovascular mortality, heart failure and stroke, but not with myocardial infarction, however in this study there was no coronary angiography performed [19].

The exact mechanisms of natriuretic peptides rise in coronary disease are not completely understood. Ischemia may constitute an independent stimulus for BNP release towards a transient decrease of systolic function and compliance, reflecting not only the impairment in left ventricular function, but also the severity of the ischemic insult [20]. Alternatively, BNP secretion may be due to the augmented regional wall stretch which occurs during ischemic attacks even in the absence of pump dysfunction inducing the neurohormonal activation [21]. The detection of BNP gene expression in ischemic and infarcted regions together with BNP receptor recruitment in coronary plaques could explain a novel mechanism of BNP induction [23,24]. The strong correlation between the Gensini Score and BNP levels extend previous findings demonstrating that a further stimulus for BNP increase could be the severity and diffusion of coronary plaques that, consistent with this observation, lead to a worsening of the ischemic myocardial area $[21,22]$. All together these data could explain the mechanisms linking BNP to an adverse outcome in CAD: it represents a marker of coronary disease severity and is related to the presence of plaques diffusion and narrowing. For the above mentioned reasons BNP should be considered as an indicator of regional ischemia and as a predictor for adverse events in patients with chest pain $[25,26]$.

\section{Study limitations}

The present study suffers from several limitations, the major one is the small sample size. However our sample was carefully defined and selected on the basis of echocardiographic and clinical criteria. Our results may have been partially influenced by a diastolic dysfunction that was not reported in all patients. Age and sex could have influenced the findings even if the population studied had similar demographic characteristics in all groups. We did not compare BNP levels to other biomarkers of myocardial injury such as Troponin, high sensitive CRP or cytokine levels. At last, the study was a retrospective analysis: the results should be confirmed in a prospective study with a larger population.

\section{Conclusions}

BNP represents a biomarker for left ventricular dysfunction and enlargement as well as for myocardial ischemia. It appears able to give additional prognostic information to existing traditional biomarkers (i.e. troponin and C-reactive protein). BNP is a candidate for entry into the setting of principal risk scores. Our findings indicate that the level of BNP may reflect the extent or severity of ischemic insult even when irreversible injury and systolic dysfunction have not occurred. 


\section{Acknowledgement}

We are grateful to Inverness Medical for the supply of the BNP laboratory kit. We thank Dr. Nancy Saymour for the technical assistance and for the help in revising the manuscript.

\section{References}

[1] Levin ER, Gardner DG, Samson WK. Natriuretic peptides. [Review]. N Engl J Med 1998;339:321-8.

[2] Arakawa N, Nakamura M, Aoki H, Hiramori K. Plasma brain natriuretic peptide concentrations predict survival after acute myocardial infarction. J Am Coll Cardiol 1996;27:1656-61.

[3] de Lemos JA, Morrow DA, Bentley JH, et al. The prognostic value of B-type natriuretic peptide in patients with acute coronary syndromes. $\mathrm{N}$ Engl J Med 2001:345:1014-21.

[4] Morrow DA, De Lemos JA, Sabatine MS, et al. Evalutation of B-type natriuretic peptide for risk assessment in unstable angina/non-ST elevation myocardial infarction. J Am Coll Cardiol 2003;41:1264-72.

[5] Antman EM, Cohen M, Bernink PJ, et al. The TIMI risk score for unstable angina/ non ST elevation MI: a method for prognostication and therapeutic decision making. JAMA 2000;284:835-42.

[6] Galvani M, Ottani F, Oltrona L, et al. N-terminal pro-brain natriuretic peptide on admission has prognostic value across the whole spectrum of acute coronary syndromes. Circulation 2004;13(110):128-34.

[7] Bazzino O, Fuselli JJ, Botto F, et al. Relative value of $\mathrm{N}$-terminal probrain natriuretic peptide, TIMI risk score, ACC/AHA prognostic classification and other risk markers in patients with non-ST elevation coronary syndromes. Eur Heart J 2004;25: 859-66.

[8] ACC/AHA 2007 Guidelines for the Management of Patients With Unstable Angina/NonST-Elevation Myocardial Infarction A Report of the American College of Cardiology/ American Heart Association Task Force on Practice Guidelines (Writing Committee to Revise the 2002 Guidelines for the Management of Patients With Unstable Angina/NonST-Elevation Myocardial Infarction) Developed in Collaboration with the American College of Emergency Physicians, the Society for Cardiovascular Angiography and Interventions, and the Society of Thoracic Surgeons Endorsed by the American Association of Cardiovascular and Pulmonary Rehabilitation and the Society for Academic Emergency Medicine. J Am Coll Cardiol 2007;50:e1-e157.

[9] Alpert JS, Thygesen K, Antman E, Bassand JP. Myocardial infarction redefined- a consensus document of the Joint European Society of cardiology/American College of Cardiology Committee for the redefinition of myocardial infarction. J Am Coll Cardiol 2000;36:959-69.

[10] Schiller NB, Shah PN, Crawford M, et al. Recommendations for quantification of the left ventricle by two-dimensional echocardiography. J Am Soc Echocardiogr 1989;2:358-67.
[11] Gibson CM, Ryan KA, Kelley M, et al. Methodologic drift in the assessment of TIMI grade 3 flow and its implications with respect to the reporting of angiographic trial results. The TIMI Study Group. Am Heart J 1999;137:1179-84.

[12] Gensini GG. A more meaningful scoring system for determining the severity of coronary heart disease. Am J Cardiol 1983;51:606.

[13] Omland T, Persson A, Ng L, et al. N-terminal pro-B-type natriuretic peptide and long-term mortality in acute coronary syndromes. Circulation 2002;106:2913-8.

[14] Sadanandan S, Cannon CP Chekuri $K$, et al. Association of elevated B-type natriuretic peptide levels with angiographic findings among patients with unstable angina and non-ST-segment elevation myocardial infarction. J Am Coll Cardiol 2004;44:564-8.

[15] Sabatine MS, Morrow DA, de Lemos JA, et al. Multimarker approach to risk stratification in non-ST elevation acute coronary syndromes. Circulation 2002;105:1760-3.

[16] Omland T, Aakvaag A, Bonarjee VV, et al. Plasma brain natriuretic peptide as an indicator of left ventricular systolic function and long-term survival after acute myocardial infarction: comparison with plasma atrial natriuretic peptide and N-terminal proatrial natriuretic peptide. Circulation 1996;93:1963-9.

[17] Palazzuoli A, Deckers J, Calabrò A, et al. Brain Natriuretic Peptide and Other Risk Markers for Outcome Assessment in Patients With Non-ST-Elevation Coronary Syndromes and Preserved Systolic Function. Am J Cardiol 2006;98:1322-8.

[18] Weber M, Dill T, Arnold R, Rau M, Ekinci O, et al. N-terminal B-type natriuretic peptide predicts extent of coronary artery disease and ischemia in patients with stable angina pectoris. Am Heart J 2004;148:612-20.

[19] Omland T, Sabatine MS, Jablonski KA, Rice MM, Hsia J, et al. Prognostic value of B-Type natriuretic peptides in patients with stable coronary artery disease. J Am Coll Cardiol 2007;50:205-14.

[20] Marumoto K, Hamada M, Hiwada K. Increased secretion of atrial and brain natriuretic peptides during acute myocardial ischaemia induced by dynamic exercise in patients with angina pectoris. Clin Sci (Colch) 1995;88:551-6.

[21] Staub D, Nusbaumer C, Zellweger MJ, et al. Use of B-type natriuretic peptide in the detection of myocardial ischemia. Am Heart J 2006;151:1223-30.

[22] Burley DS, Hamid SA, Baxter GF. Cardioprotective action of peptide hormones in myocardial ischemia. Heart Fail Rev 2007;12:279-91.

[23] Ndrepepa G, Braun S, Shomig A, Kastrati A. Accuracy of N-terminal Pro-brain Natriuretic Peptide predict mortality in various subset of patients with coronary artery disease. Am J Cardiol 2007; 100:575-8.

[24] Barbato E, Rubattu S, Bartunek J, Berni A, Sarno G, et al. Human coronary atherosclerosis modulates cardiac natriuretic peptide release. Atherosclerosis 2009;206(1):258-64.

[25] Bassan R, Potsch A, Maisel A, Tura B, Villacorta H, Nogueira MV, Campos A, Gamarski R, Masetto AC, Moutinho MA. B-type natriuretic peptide: a novel early blood marker of acute myocardial infarction in patients with chest pain and no ST-segment elevation. Eur Heart J 2005;26:234-40.

[26] Kwan G, Isakson SA, Beede J, Clopton P, Maisel A, et al. Short-term serial sampling of natriuretic peptides in patients presenting with chest pain. J Am Coll Cardiol 2007:49:1186-92. 